\title{
Multiaxial fatigue strength under non- proportional loading of additively manufactured notched components of Ti-6Al-4V
}

\author{
Stefano Bressan ${ }^{1}$, Javad Razavi $^{2}$, Fumio Ogawa ${ }^{3}$, Takamoto Itoh $^{3}$ and Filippo Berto ${ }^{2}$ \\ ${ }^{1}$ Ritsumeikan University, Graduate School of Science \& Engineering, 1-1-1, Kusatsu-shi, Shiga, \\ 5258577, Japan \\ ${ }^{2}$ Department of Mechanical and Industrial Engineering, Norwegian University of Science and \\ Technology (NTNU), Richard Birkelands vei 2b, 7491, Trondheim, Norway \\ ${ }^{3}$ Ritsumeikan University, Department of Mechanical Engineering, 1-1-1, Kusatsu-shi, Shiga, 5258577, \\ Japan
}

\begin{abstract}
In this study fatigue tests have been conducted on additively manufactured notched specimens made of titanium alloy Ti-6Al-4V under uniaxial loading and multiaxial non-proportional loading. Laser powder bed fusion technique has been employed to fabricate cylindrical specimens which have been successively machined to obtain a circumferential notch. Four varieties of specimens were fabricated depending on the build orientation and the application of a post-fabrication stress-relief heat treatment. The influence of layer orientation and heat treatment on the material microstructure has been analyzed. Strain controlled tests were performed with both proportional loading represented by a push-pull strain path and non-proportional loading represented by a circle strain path. The number of cycles to failure under both proportional and non-proportional loadings seemed not depending on layer orientation and heat-treatment. The cyclic plastic behavior of the material has been verified through the observation of the softening and hardening curves. The surface of the crack has been finally analyzed to verify the crack initiation position which has been detected on voids or defects located in the vicinity of the notch tip.
\end{abstract}

\section{Introduction}

In the field of material innovation, additive manufacturing (also known as 3D printing) of metals represents the latest trend of research [1,2]. Additive manufacturing or AM refers to a fabrication technique for creating parts layer by layer through different techniques. The available techniques nowadays can be broadly divided in powder bed fusion technique and direct energy deposition techniques. Powder bed fusion techniques consists in melting a thin layer of powder, previously deposited on a moving platform using laser or electron beam as power source. After one layer is created, the moving platform lowers, allowing the deposition of new powder and creation of the next layer. The direct energy deposition system is a technique employing powder injection or wire feed into a molten pool created by a focused, high-powered laser beam. Although additive manufacturing introduces a series of advantages such as the high fabrication speed and the possibility of manufacturing components with complicated shapes. However, the components produced by additive manufacturing techniques commonly suffer from high surface roughness, presence of

Corresponding author: gr0300kf@ed.ritsumei.ac.jp 
residual stresses and internal defects such as voids and lack of fusions $[3,4]$. To avoid the aforementioned drawbacks, several countermeasures such as heat treatments and surface machining can be adopted to improve mechanical characteristics of the material.

The major challenge in the definition of the static and fatigue characteristics of materials fabricated with such techniques is the wide variety of microstructures obtainable due to the wide range of process parameters that can be set in the in the fabrication phase. Despite this obstacle, several researches have been conducted and a common trend in the mechanical properties in relation to the microstructure have been defined [5,6]. In terms of fatigue properties, the work presented by Li et. al evidenced that surface roughness exerts the major influence on fatigue life, followed by voids and layer orientation [7]. The fatigue properties have been investigated mainly by testing samples under uniaxial high cycle fatigue. In practical applications, components' geometrical discontinuity features represented by notches, grooves and holes that provoke phenomena of stress concentration may lead to local plasticity. Furthermore, such components are often subjected to multiaxial loading conditions (proportional or non-proportional) which further complicates the mechanical response of the material. In a previous work, the influence of heat treatment and layer orientation on uniaxial and multiaxial non-proportional low cycle fatigue have been investigated [8] and the heat-treatment has been found having the major influence on fatigue life compared to the layer orientation and voids. In the current literature, there are few works discussing the low cycle fatigue of AM notched components made of Ti-6Al-4V and further tests are necessary to define the material behavior also in relation to geometrical discontinuities.

The present work aims to investigate the low cycle fatigue behavior of four varieties of AM notched specimens. The microstructure has been analyzed to verify the microstructural differences between the varieties. The stress-strain cyclic curves have been obtained for each specimen to verify the cyclic plastic response of the material. Fatigue tests have been conducted, and the difference in terms of fatigue life between the specimen varieties are discussed. Crack initiation site has been observed on the fracture surface, focusing on the observation of cracks initiating from internal defects and their position on the notch surface.

\section{Specimens and materials properties}

\subsection{Specimens}

The specimens have been fabricated employing the L-PBF (Laser Power Bad Fusion) fabrication technique. The specimens have been successively machined to obtain the final shape of the specimens (Fig.1 a). The notch geometry has been designed to obtain a blunt notch with the stress concentration factor of $K_{\mathrm{t}, \mathrm{n}}=1.5$ referred to the net section.

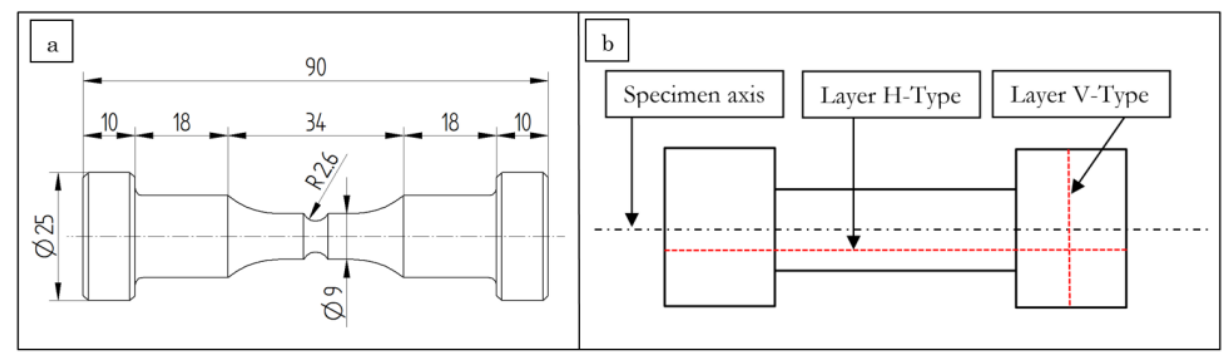

Fig. 1. Specimens geometry (a) and schematic view of the layer orientations (b). 
The varieties of specimens depended on the layer orientation and the presence of a stress-relief heat treatment. The layer orientation was defined horizontal and vertical according to Fig. 1 b. Stress relief heat treatment was conducted at $800{ }^{\circ} \mathrm{C}$ for 4 hours. The specimens have been named based on the layer orientation and heat treatment: HT (horizontal, heat-treated), HNT (horizontal, non-heat-treated), VT (vertical, heat-treated), and VNT (vertical, non-heat-treated).

\subsection{Microstructure}

The microstructure of each variety of specimens have been observed and presented in Figs. $2 \mathrm{a}$ and $\mathrm{b}$. For the sake of brevity, only the microstructures of the H-specimens are shown.

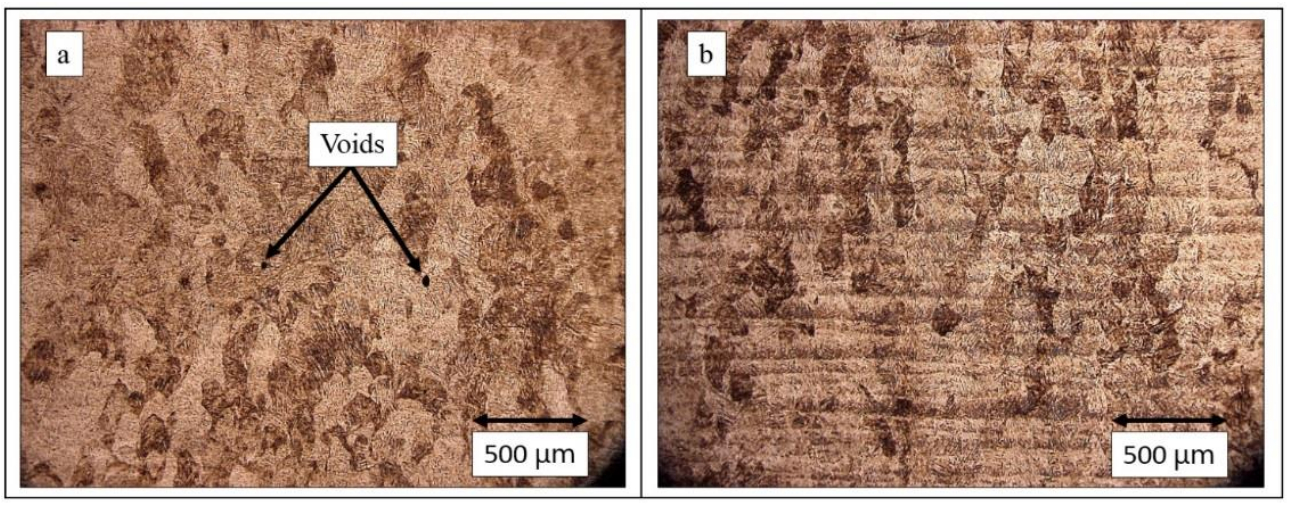

Fig. 2. Material microstructure of HT (a) and HNT (b) type specimens.

The performed heat treatment for releasing the residual stresses is not such as to induce phase transformation, grain coarsening, etc. The elongated prior $\beta$-grains are visible in the form of dark and light areas, which depend on the crystal orientation. The direction of the prior $\beta$-grains depends on the layer orientation and the average length of the grain was measured $200 \mu \mathrm{m}$. The axis of the elongated prior $\beta$-grains is perpendicular to the layer. The heat flow direction is perpendicular to the layer which consequently results in the grain growth direction perpendicular to the layer. Voids with the average size of $30 \mu \mathrm{m}$ have been detected in each specimen. The voids were spherical and isolated. The void density and dimension were independent of heat treatment and layer orientation.

\subsection{Cyclic stress-strain curves}

The cyclic plastic properties have been obtained with both push-pull tests (PP) and 90 degrees out-of-phase axial-torsional non-proportional strain path called circle (OP) to investigate the cyclic plastic behavior of each specimen (Fig. 3). The cyclic curves have been obtained with the step-up method, consisting in increasing the applied strain range by $0.1 \%$, from 0 to $2 \%$ every 10 cycles. The cyclic stress strain curves are represented in Figs. $4 \mathrm{a}$ and $\mathrm{b}$.

The cyclic stress-strain curves show that the plastic cyclic behavior of different types of specimens presents many similarities. In the case of PP strain path, the heat-treated samples exhibited slightly higher elastic modulus $E$ compared to the non-heat-treated samples. In 
case of OP, the elastic modulus appears to be the same for each tested specimen. The plastic trait does not suggest any tendency of softening or hardening both for PP and OP. In case of OP the transition between elastic and plastic behavior occurs earlier compared to the PP case.

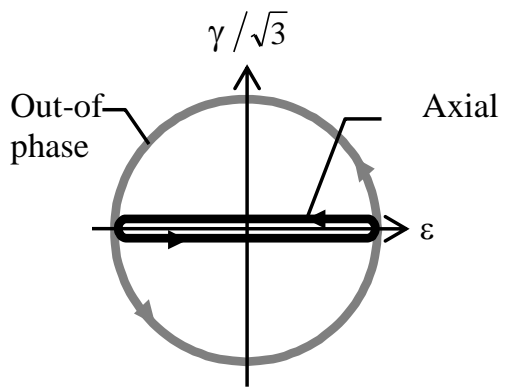

Fig. 3. Representation of the applied strain paths
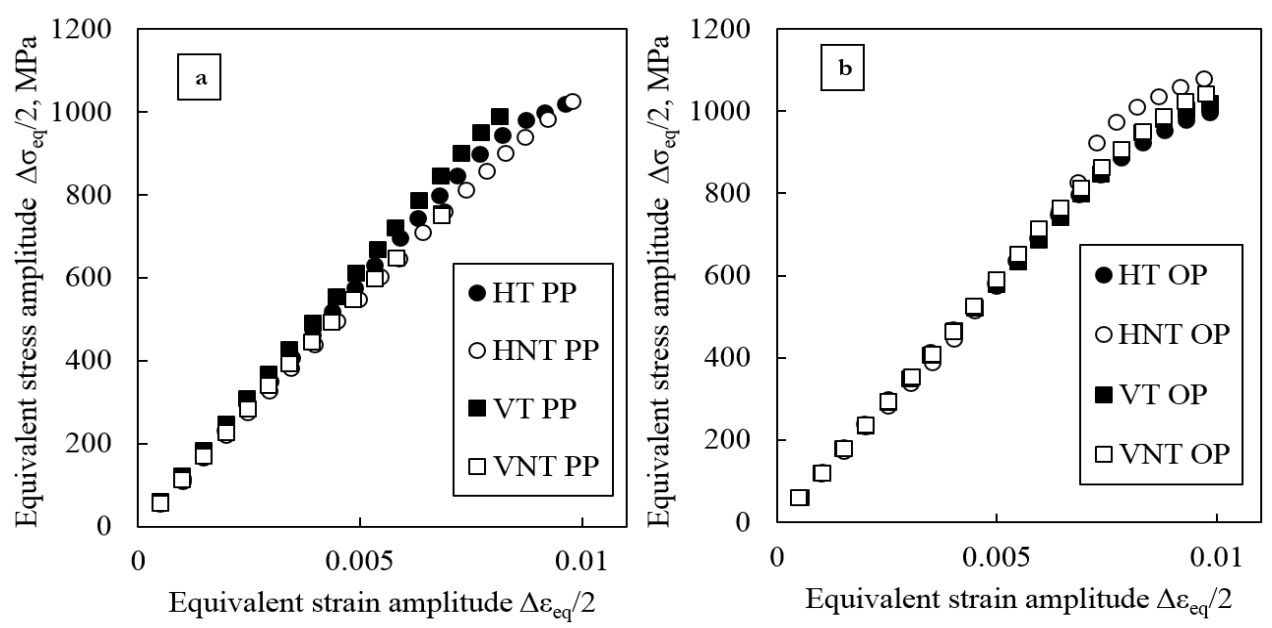

Fig. 4. Cyclic stress-strain curves for PP (a) and OP (b) strain paths.

\section{Fatigue tests}

\subsection{Fatigue tests conditions}

The fatigue tests have been conducted with the same test machine employed to obtain the stress-strain cyclic curves. The tests were strain-controlled with a gage length of $12 \mathrm{~mm}$. von Mises equivalent strain range $\Delta \varepsilon_{\mathrm{eq}}=0.7 \%$ has been applied for uniaxial and multiaxial loadings to induce a localized plastic deformation in the vicinity of the notch. The number of cycles to failure (failure life, $N_{\mathrm{f}}$ ) was defined as the number of cycles at which the recorded von Mises equivalent nominal stress at the net section of the specimen $\Delta \sigma_{\text {eq, }} / 2$ became equal to $3 / 4$ of the maximum von Mises equivalent nominal stress recorded in the cycle.

\subsection{Fatigue tests results}


The fatigue tests have been conducted with the same test machine employed to obtain the cyclic stress-strain curves. The results of the fatigue life tests are represented in Table 1.

Table 1. Fatigue test results

\begin{tabular}{|c|c|c|c|c|}
\hline Specimen Type & Strain Path & $\boldsymbol{\Delta} \boldsymbol{\sigma}_{\mathbf{n}, \mathbf{m a x} / \mathbf{2}(\mathbf{M P a})}$ & $\boldsymbol{\Delta} \boldsymbol{\tau}_{\mathbf{n}, \mathbf{m a x}} / \mathbf{2}(\mathbf{M P a})$ & $\begin{array}{c}\text { Fatigue } \\
\text { Life }\end{array}$ \\
\hline HT & PP & 640 & - & 12027 \\
\hline HNT & PP & 600 & - & 10022 \\
\hline VT & PP & 640 & - & 7771 \\
\hline VNT & PP & 637 & - & 10297 \\
\hline HT & CI & 630 & 205 & 1774 \\
\hline HNT & CI & 630 & 230 & 1668 \\
\hline VT & CI & 720 & 220 & 1163 \\
\hline VNT & CI & 950 & 230 & 983 \\
\hline
\end{tabular}

The fatigue life of the specimens appears to be not dependent on layer orientation or heat treatment except for the case of multiaxial loading. In case of push-pull strain path, the fatigue life of the heat-treated components with horizontal layer is higher than the not heattreated counterpart. However, in case of vertical layer, the non-heat-treated specimen exhibits the longest life. Therefore, a correlation between layer orientation, heat-treatment and failure life could not be defined. In case of out-of-phase strain path, the overall fatigue life is $10 \%$ of the one resulted from the uniaxial tests. The V-notched specimen exhibits a shorter fatigue life compared to the one of the H-specimens. The initial levels of maximum axial stress and shear stress $\left(\Delta \tau_{n, \max } / 2\right)$ are similar for each specimen variety.

\section{Discussion}

\subsection{Hardening and softening curves}

The hardening and softening curves have been obtained for both the uniaxial and multiaxial tests and they are represented in Figs. 5a and b. The nominal stress is averagely 620MPa, meaning that the stress at the notch tip becomes approximately $930 \mathrm{MPa}$ which provokes a deformation in the plastic field, according to the cyclic curves reported in Fig. 4. In case of PP, no hardening or softening have been observed. In fact, the stress levels remain constant before a sudden drop which coincide with the crack propagation leading to the final failure. The slope of the final trait is slightly different between the vertical and horizontal specimens, indicating that the final crack propagation mechanism is different as reported also in [8]. In case of OP, an anomalous hardening behavior have been observed for the vertical specimens and the causes are still under investigation and may represent the cause of the earlier failure life exhibited by the vertical layered specimens in case of multiaxial loading. 

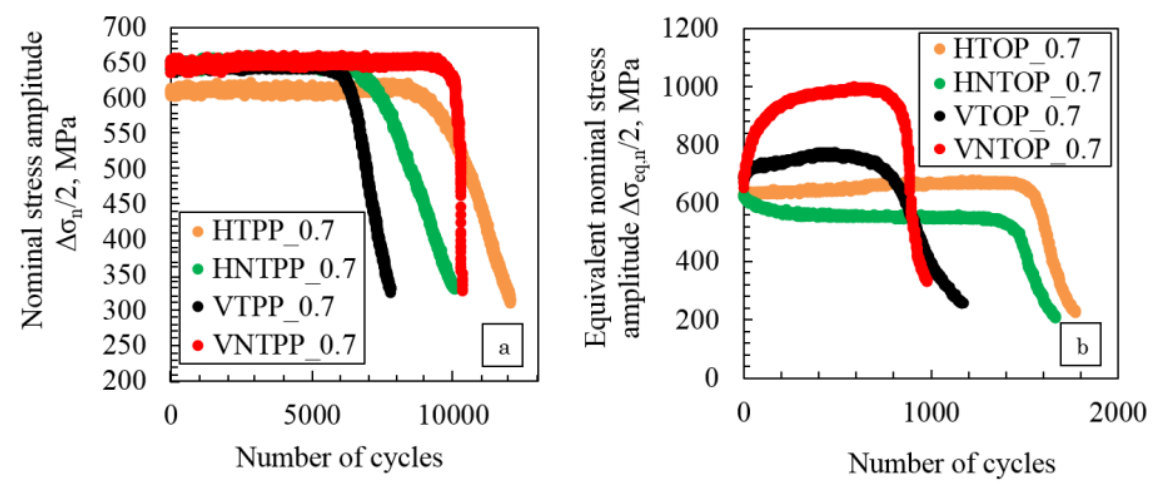

Fig. 5. Softening and hardening curves in case of PP (a) and OP (b)

\subsection{Fracture surface}

The specimens fracture surfaces have been analyzed with a scanning electron microscope (SEM) to further investigate the crack initiation sites. Some of the most relevant observations are reported in Fig. 6.
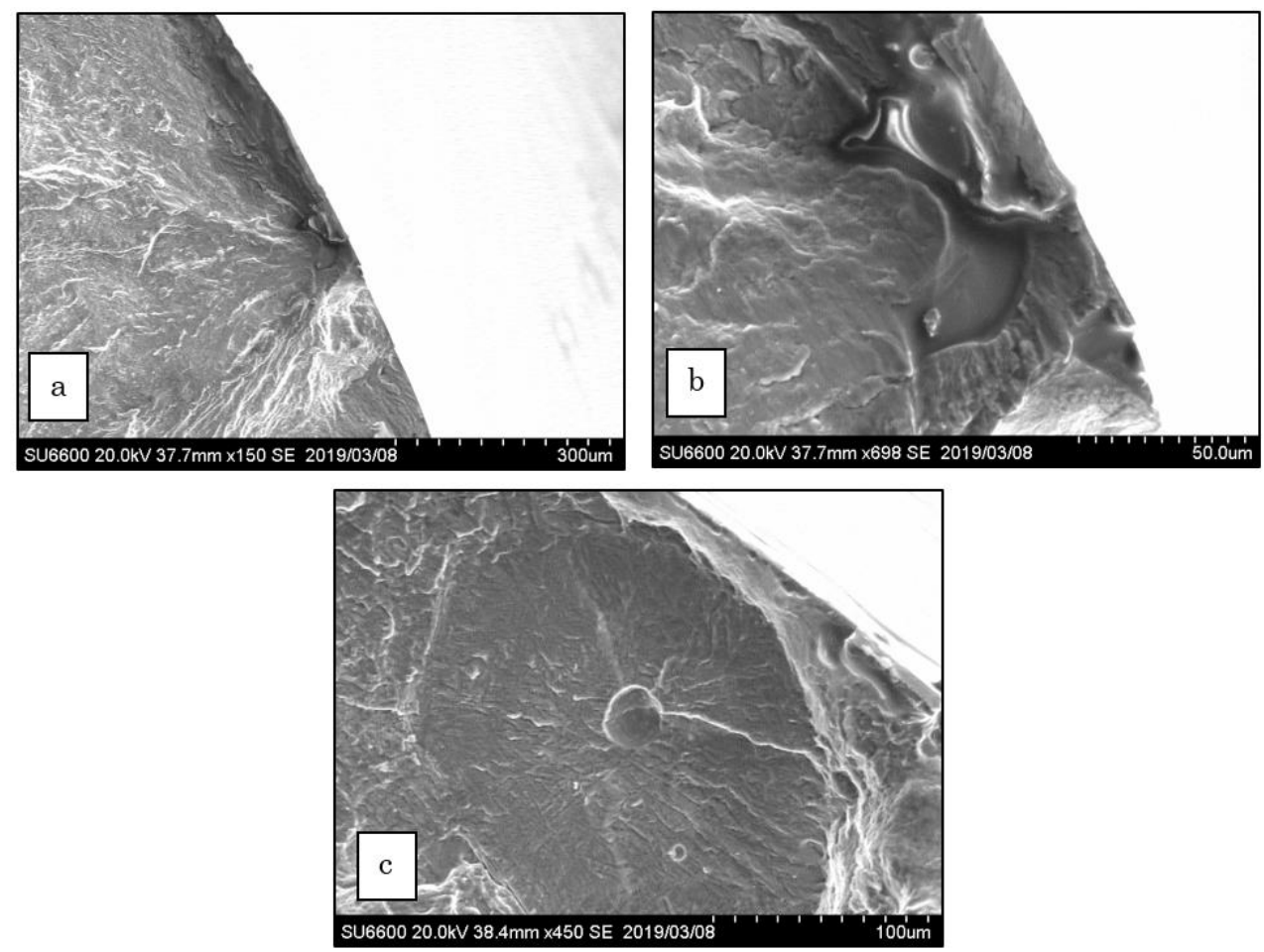

Fig. 6. SEM pictures of crack initiating from the inner defect (a), detail of the defect (b), crack initiating from an internal void (c)

In each analyzed case, the crack initiation site was located on the notch tip. The crack initiation site was located in the proximity of the circular notch, and the initiation occurred 
under different modalities. The SEM observation are representative of both cases of uniaxial and multiaxial strain path. In Figs. 6a and 6b (detail of a), the crack initiation site was located on an internal lack of fusion type of defect. In this case, in fact, no crack on the notched surface could be detected after the test. Furthermore, no other crack initiation site could be detected on this specimen, meaning that the failure life was driven by the presence of the lack of fusion type of defect. Cracks initiating from internal voids located in the vicinity of the notch tip could also be detected (Fig. 6 c). It is still unclear whether such cracks represent the initiation site of the crack which propagate and led to failure. A correlation between the modality crack initiation and layer orientation or heat-treatment is currently under investigation.

\section{Conclusions}

In order to verify the uniaxial and multiaxial low cycle fatigue behavior of four varieties of AM Ti-6Al-4V specimens and the influence of the geometrical discontinuities, notched specimens have been tested with strain-controlled fatigue test. The conclusion of this work can be summarized as follows.

1. Failure life of notched specimens seems not having a correlation with layer orientation and heat-treatment, although an anomalous hardening on the multiaxial tests of vertical specimens might be the cause for the earlier failure life.

2. In the case of PP, the final crack propagation rate could be connected to the layer orientation as previously observed on smooth specimens made of the same material [8].

3. The crack initiation modalities were mainly from lack of fusion between the layers and internal voids. All the crack initiation sites were located at the vicinity of the notch tip.

\section{References}

1. J.P. Kruth, MC. Leu, T. Nakagawa. Progress in additive manufacturing and rapid prototyping. CIRPAnn 47: 525-540 (1998) doi:10.1016/S0007-8506(07)63240-5.

2. G.N. Levi, R. Schnidel, J.P. Kruth. Rapid manufacturing and rapid tooling with layer manufacturing (LM) technologies, state of art and future perspectives. CIRP Ann 52: 589-609 (2003) doi:10.1016/S0007-8506(07)60206-6

3. M. Shiomi, K. Osakada, K. Nakamura, T. Yamashita, F. Abe. Residual stress within metallic model made by selective laser melting process. CIRP Ann 53: 195-198 (2004) doi:10.1016/S0007-8506(07)60677-5.

4. B. Baufeld, E. Brandl, O. van der Biest. Wire Based Additive Layer Manufacturing: Comparison of Microstructure and Mechanical Properties of Ti-6Al-4V Components Fabricated by Laser-Beam Deposition and Shaped Metal Deposition. J. Mater. Process. Technol. 211: 1146-58 (2011) doi:10.1016/j.jmatprotec.2011.01.018.

5. X. Tan, Y. Kok, Y.J. Tan, M. Descoins, D. Mangelinck, S.B Tor et al. Graded microstructure and mechanical properties of additive manufactured $\mathrm{Ti}-6 \mathrm{Al}-4 \mathrm{~V}$ via electron beam melting. Acta Mater. 97: 1-16 (2015) doi:10.1016/j.actamat.2015.06.036. 
6. C. Qiue, N.J.E Adkinsm, M.M Attallah, Microstructure and tensile properties of selectively laser-melted and of HIPed laser-melted Ti-6Al-4V. Mater. Sci. Eng. A. 578: 230-239 (2013) doi:10.1016/j.msea.2013.04.099.

7. P. Li, D.H. Warner, A. Fatemi, N. Phan. Critical assessment of the fatigue performance of additively manufactured $\mathrm{Ti}-6 \mathrm{Al}-4 \mathrm{~V}$ and perspective for future research. Int. J. Fatigue 85: 130-143 (2016) doi:10.1016/j.ijfatigue.2015.12.003.

8. S. Bressan, F. Ogawa, T. Itoh, F. Berto. Low cycle fatigue behavior of additively manufactured Ti-6Al-4V under non-proportional and proportional loading. Frattura integr. strutt. 48: 18-25 (2019) 